\title{
Diagnosis of extrapulmonary tuberculosis using the MPT64 antigen detection test in a high-income low tuberculosis prevalence setting
}

Ida Marie Hoel ${ }^{1,2,3^{*}}$ (D) Lisbet Sviland ${ }^{3,4}$, Heidi Syre ${ }^{5}$, Anne Ma Dyrhol-Riise ${ }^{2,6,7}$, Ingerid Skarstein ${ }^{8}$, Peter Jebsen ${ }^{9}$, Melissa Davidsen Jørstad ${ }^{1,10}$, Harald Wiker ${ }^{2}$ and Tehmina Mustafa ${ }^{1,10}$

\begin{abstract}
Background: Extrapulmonary tuberculosis (EPTB) poses diagnostic challenges due to the paucibacillary nature of the disease. The immunochemistry-based MPT64 antigen detection test (MPT64 test) has shown promising results for diagnosing EPTB in previous studies performed in low-resource settings, with higher sensitivity than microscopy and culture. The aim of this study was to investigate the performance of the MPT64 test in a routine clinical setting in a high-income low TB prevalence country.
\end{abstract}

Methods: Extrapulmonary samples sent for TB diagnostics to microbiology and pathology laboratories at three regional tertiary care hospitals in Norway in a one-year period were included and subjected to the MPT64 test in parallel to the routine TB diagnostic tests.

Results: Samples from 288 patients were included and categorised as confirmed TB cases $(n=26)$, clinically diagnosed TB cases $(n=5)$, non-TB cases $(n=243)$ and uncategorised $(n=14)$, using a composite reference standard (CRS). In formalin-fixed biopsies, the sensitivity (95\% CI) of the MPT64 test, microscopy, PCR-based tests pooled, and culture was 37\% (16-62), 20\% (4-48), 37\% (16-62) and 50\% (23-77), respectively, against the CRS. The MPT64 test showed a good positive predictive value (88\%) and an excellent specificity $(99,95 \% \mathrm{Cl} 92-100)$ in formalin-fixed biopsies. In fine-needle aspirates, pus and fluid samples, the test performance was lower.

Conclusions: The MPT64 test was implementable in pathology laboratories as part of routine diagnostics, and although the sensitivity of the MPT64 test was not better than culture in this setting, the test supplements other rapid diagnostic methods, including microscopy and PCR-based tests, and can contribute to strengthen the diagnosis of EPTB in formalin-fixed biopsies in the absence of culture confirmation.

Keywords: Extrapulmonary tuberculosis, Diagnostic test, Immunohistochemistry, MPT64, Antigen detection test

\section{Background}

While tuberculosis $(\mathrm{TB})$ remains a global health problem, the incidence in Norway and many other high-income countries is low [1]. Still, diagnosis and control of TB disease poses significant challenges in high-income settings. Although TB rates have been continuously declining in

\footnotetext{
* Correspondence: Ida.Hoel@uib.no

${ }^{1}$ Centre for International Health, Department of Global Public Health and Primary Care, University of Bergen, Bergen, Norway

${ }^{2}$ Department of Clinical Science, University of Bergen, Bergen, Norway

Full list of author information is available at the end of the article
}

the Norwegian-born population since the middle of the past century, the overall TB incidence in Norway and other high-income countries has remained relatively stable over the last years because of immigration from TB prevalent countries [2-5]. Several studies also report that the increase in foreign-born $\mathrm{TB}$ cases is associated with a rise in the proportion of extrapulmonary TB (EPTB) [3, 5-7]. In the European region, EPTB has increased from 16,4\% of all TB cases in 2002 to $22,8 \%$ in 2016 [6, 8]. In the Netherlands, England, Australia and Norway, EPTB currently accounts for as much as $40 \%$ of all TB cases $[1,2]$. 
The diagnosis of EPTB is challenging. Clinical and radiological findings are often non-specific and the sensitivity of routine TB diagnostic tests, including microscopy for acid fast bacilli (AFB) and culture, is low in paucibacillary disease [9]. Culture also requires advanced laboratory facilities, and results could be delayed up to 8 weeks. Globally, the use of rapid molecular tests for detection of $\mathrm{TB}$ is increasing, albeit most commercially available PCR-based tests are only approved for pulmonary TB. The only World Health Organization (WHO) endorsed PCR-based test for diagnosing EPTB, Xpert MTB/RIF (Cepheid, Sunnyvale, CA), has shown variable sensitivity in extrapulmonary samples [10] and is only recommended for subgroups of EPTB [11]. A recently launched new version, Xpert MTB/RIF Ultra (Xpert Ultra), performs better in smear negative, culture positive sputum samples [12], but so far, few studies have investigated its use in EPTB [13-18]. Histopathological findings suggestive of TB may support the EPTB diagnosis, but these are also present in other diseases including sarcoidosis and non-tuberculosis mycobacteria (NTM) infections. The incidence of NTM infections is also increasing in western countries [19-22]. Due to these diagnostic challenges, a definite diagnosis of EPTB is often difficult to obtain. Many EPTB patients are diagnosed clinically and EPTB is associated with diagnostic delay [23-25]. Thus, better diagnostic tests are needed to improve early case detection and management of EPTB patients.

An immunochemistry-based test for detection of the mycobacterial secreted protein MPT64 (MPT64 test) from biopsies, fine-needle aspirates (FNAs) and fluid samples has shown high sensitivity for diagnosing ЕРТВ in previous studies compared to culture and a TB specific nested-PCR [26-31]. The MPT64 test is robust and fast, and can differentiate between NTM and TB disease, as the MPT64 protein is specific for Mycobacterium tuberculosis complex (MTBC) species, and not found in NTM [32-34]. A recent study conducted in Zanzibar, Tanzania, has also shown that the MPT64 test is implementable in a routine $\mathrm{TB}$ diagnostic setting in a TB high-endemic low-resource country [35]. However, the performance of the MPT64 test has not yet been evaluated in a routine clinical setting in a low TB burden high-income country. The objective of the study was to evaluate performance of the MPT64 test and whether the test would provide an added value to EPTB diagnostics when implemented in routine TB diagnostics in the high-resource health care system in Norway.

\section{Methods}

\section{Sample inclusion}

Formalin-fixed biopsies, FNAs and fluid samples sent for TB diagnostics to microbiology and pathology laboratories at three regional tertiary care hospitals (Haukeland University Hospital (HUH), Oslo University Hospital (OUH) and Stavanger University Hospital (SUH)) from January 2015 until January 2016 were prospectively included in the study, provided there was enough material left after routine diagnostics to prepare a minimum of one cell smear or tissue section for the study (Fig. 1). Acellular fluid samples and all samples from patients that had received TB treatment during one year prior to the study, were excluded.

Because very few formalin-fixed biopsies from patients with presumptive TB had been prospectively included, a retrospective inclusion of biopsy specimens was also performed. At $\mathrm{HUH}$ and $\mathrm{SUH}$, all samples included in the study from the microbiology laboratories were crosschecked with the pathology laboratory registers to see if the same sample, or a different sample material collected from the same location at the same time, had been sent to the pathology laboratories. At OUH, a list of all biopsies sent for TB diagnostics to the microbiology laboratory during 2015 was cross-checked with the pathology register to find the samples that had been sent for both departments. Based on these searches, formalin-fixed biopsies from the pathology departments were included if they showed any type of inflammation or necrosis. Biopsies with a histopathological diagnosis other than TB (e.g. malignancy) or no pathological findings were not included as these samples will not be subjected to TB specific tests at the pathology laboratory in a routine clinical setting.. Additionally, all formalin-fixed biopsies that had been subjected to a TB specific nested-PCR as part of routine diagnostics at Department of Pathology at $\mathrm{HUH}$, were retrospectively included.

\section{Sample processing and routine TB diagnostic procedures}

All samples were subjected to routine TB diagnostics at the inclusion hospitals according to local diagnostic algorithms. At the microbiology laboratories, FNAs in saline and fluid samples were used unconcentrated if volume $<10 \mathrm{~mL}$ and concentrated by centrifugation before resuspension of sediment in saline if the sample volume was $>10 \mathrm{~mL}$. Biopsy specimens were mechanically homogenized and resuspended in saline. Cell smears were stained using the Ziehl-Neelsen or Auramine method for detection of AFB. For the study, a minimum of one cell smear from fluid samples was prepared on a Superfrost glass, air-dried for $20 \mathrm{~min}$, fixed in absolute ethanol for $20 \mathrm{~min}$ and stored at room temperature. A standard NALC-NaOH decontamination procedure was performed on the remaining sample material if the sample was non-sterile, before appropriate sample volumes were seeded in liquid medium (BACTEC MGIT), and for most of the samples, also onto solid medium (Lowenstein-Jensen). At $\mathrm{HUH}$, all lymph node specimens, sterile fluids and aspirates and most biopsies were cultured both before and after 


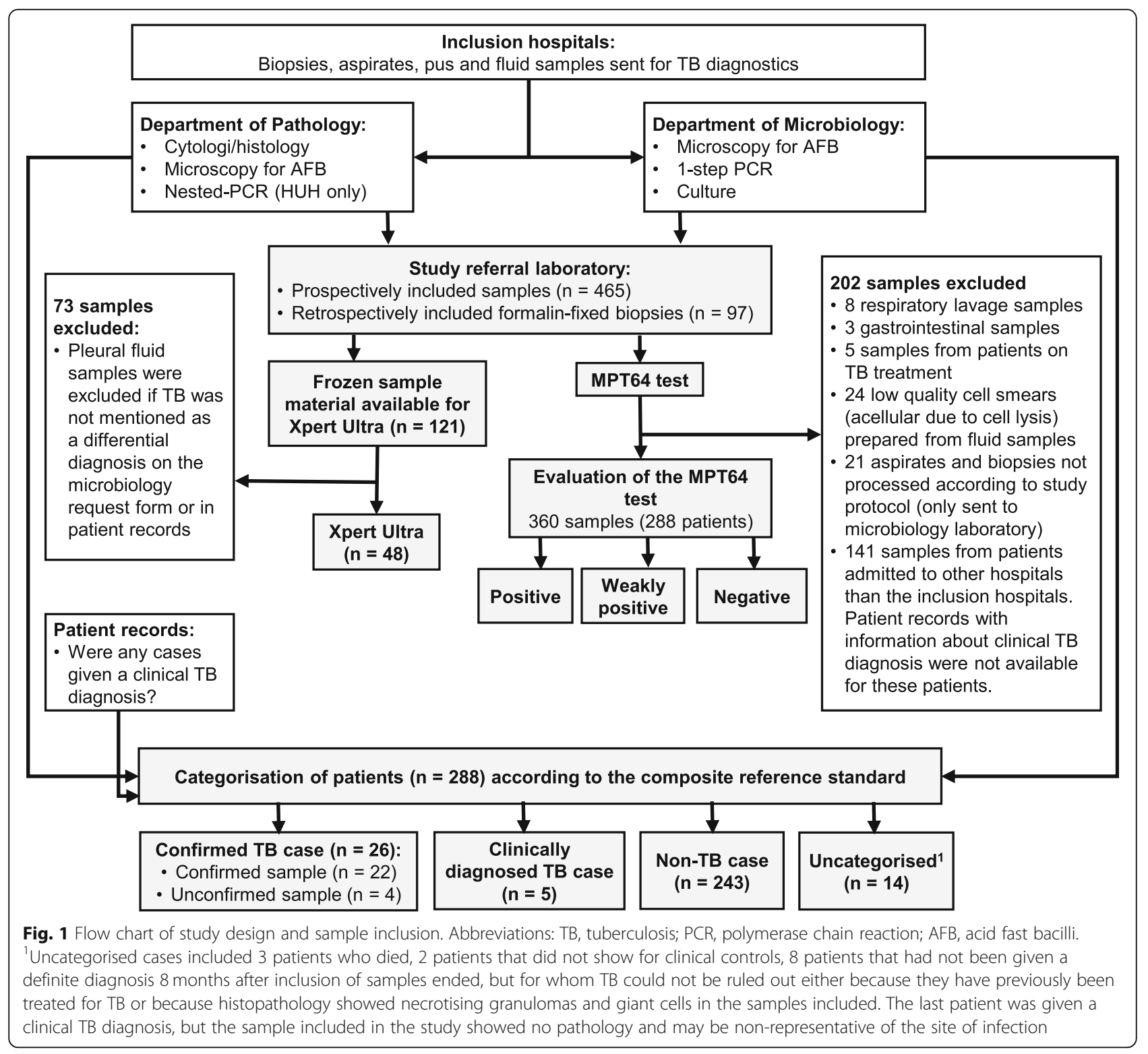

NALC-NaOH decontamination, and lymph node specimens were also cultured at $28^{\circ} \mathrm{C}$. If PCR was requested by the clinician, a 1-step PCR-based tests (1-step PCR) including Cobas Taqman MTB (Roche, Switzerland) at OUH, Abbott Real Time MTB (Abbott, United States) at SUH and Genotype MTBDR plus (Hain Lifescience, Germany) at HUH, was performed. All samples with a remaining volume of $>$ $0.5 \mathrm{~mL}$, were stored at $-80^{\circ} \mathrm{C}$ for later analysis with Xpert Ultra.

At the pathology laboratories, biopsy specimens were routinely fixed in PBS buffered formalin and embedded in paraffin before tissue sections were prepared for histology. Fine-needle aspiration from lymph nodes was performed by local clinicians or pathologists and cell smears for cytology were directly prepared and fixed after sample collection. If microscopy for AFB was requested by the pathologist, the Ziehl-Neelsen ( $\mathrm{HUH}$, SUH), Auramine (OUS) or Fite Faraco [36] (OUS) method was used. Additionally, a previously developed in-house nested-PCR (n-PCR) for detection of the MTBC-specific IS6110 sequence in DNA extracted from archived material [37] was also performed on the samples as part of routine diagnostics at HUH only, if requested by the pathologist.

\section{Xpert ultra}

Xpert Ultra was performed on all the frozen sample material during the autumn 2018, except for pleural fluid samples, which were only subjected to Xpert Ultra if TB was mentioned as a differential diagnosis on the request form or in patient records. This was done to exclude clinically irrelevant samples, as many pleural fluid samples are routinely sent for TB diagnostics, even when the 
pre-test probability of TB is very low. Samples were thawed at room temperature and processed according to the manufacturer's protocol. All but two samples (both volume $0.25 \mathrm{~mL}$ ) had a sample volume of minimum 0.5 $\mathrm{mL}$. Samples with volume $<0.7 \mathrm{~mL}(n=20)$ were added sample reagent to sample in a 3:1 ratio, whereas a ratio of 2:1 was used for samples with a volume of $0.7 \mathrm{~mL}$ or more $(n=28)$.

\section{Immunostaining with MPT64}

The MPT64 test was performed by a laboratory technician in parallel to routine TB diagnostics at Department of Pathology at HUH. The request form with clinical information, sometimes including results of $\mathrm{TB}$ diagnostic tests, was available to the technician. The test was performed using an in-house polyclonal rabbit anti-MPT64 antibody at 1:250 dilution together with the Dako Envision + System-HRP kit (Agilent, Santa Clara, CA), according to the manufacturer's protocol with some modifications as earlier described [26, 28]. Briefly, tissue sections were deparaffinized with xylene, before tissue sections and cell smears were rehydrated through decreasing grades of alcohol. Microwave antigen retrieval in citrate buffer, $\mathrm{pH}$ 6.2, was then performed on tissue sections only. Further, tissue sections and cell smears were washed in distilled water for $10 \mathrm{~min}$ and incubated with hydrogen peroxide for $20 \mathrm{~min}$. The primary anti-MPT64 antibody was applied and the slides were incubated for $60 \mathrm{~min}$, before horseradish conjugated secondary anti-rabbit antibody was applied for $45 \mathrm{~min}$. Thereafter, the substrate (3-amino-9ethylcarbazol) was added to the slides for $10 \mathrm{~min}$ for smears and $15 \mathrm{~min}$ for biopsies, followed by counterstaining with Mayer's haematoxylin and mounting with ImmuMount (Thermo Fisher Scientific, United States). Slides were washed with wash buffer $(0.05 \mathrm{~mol} / \mathrm{L}$ Tris/ $\mathrm{HCl}$ buffered saline with $0.05 \%$ Tween 20 , pH 7.6) between incubation steps.

\section{Evaluation of immunostaining}

A laboratory technologist was trained to screen the MPT64 test stained cell smears prepared from fluid samples. Screening was performed at a total magnification of 200x and more detailed evaluation at 400x. Fluid samples screened possibly positive were examined by a designated pathologist, who also evaluated all biopsies and FNAs, according to a previously developed guideline for interpretation [35]. Briefly, a sample was positive if a minimum of two granular red-brown coloured spots, either observed intracytoplasmic in inflammatory cells or extracellularly in necrotic material, were present in the sample. If only one typical spot was present, or if the staining was not strongly granular, the test was evaluated as weakly positive. No staining, nuclear staining or extracellular granular staining in non-necrotic areas were interpreted as negative. Clinical information on the request form, which sometimes included information about results of routine TB diagnostics, was available to the pathologist.

\section{Categorisation of samples and patients according to a composite reference standard}

A composite reference standard (CRS), including both microbiologically confirmed $\mathrm{TB}$ and clinically diagnosed $\mathrm{TB}$, was used to define a TB case. Results of routine TB diagnostic tests and cyto/histopathological examination were obtained from the laboratory information systems. Medical records for all patients with culture and PCR negative samples were checked for a clinical TB diagnosis 8 months after the inclusion of samples had finished. According to the CRS, a patient was defined as a confirmed TB case if a culture and/or PCR (1-step PCR and/or n-PCR) positive sample was registered during the inclusion period or on a repeat sample until 8 months afterwards. Culture and/or PCR positive samples were classified as confirmed samples from confirmed TB cases, whereas culture and PCR negative samples from patients that were diagnosed with microbiologically confirmed TB within 8 months after end of inclusion, were classified as unconfirmed samples from confirmed TB cases. A patient that had been given a clinical TB diagnosis and successfully completed a full course of TB treatment, was defined as a clinically diagnosed TB case. Patients with culture and PCR negative samples that improved without treatment, or were given a diagnosis other than $\mathrm{TB}$, or had a negative interferon-gamma-release assay, or had stable symptoms and negative results of TB diagnostics at repeated controls until 8 months after the inclusion had finished, were defined as non-TB cases. Patients that did not fit into any of these categories were classified as uncategorised cases.

\section{Statistical analysis}

Test performance was evaluated using one sample per case. When multiple samples were included from one case, the first sample collected from non-TB cases and the first confirmed TB sample collected from TB cases was chosen for analysis. For TB cases with multiple unconfirmed samples included, the sample with cyto/ histopathological findings most strongly suggestive of TB was chosen. Sensitivity, specificity and accuracy were calculated using the CRS as reference method.

\section{Results}

\section{Clinical samples}

A total of 465 samples received for TB diagnostics at the inclusion hospitals were consecutively sent to the $\mathrm{HUH}$ during the study period (Fig. 1). Additionally, 97 samples were retrospectively included from the same hospitals. 
After exclusion of 202 samples for various reasons, the remaining 360 samples from 288 patients were classified using the CRS. Twenty-six patients were confirmed TB cases, 5 clinically diagnosed TB cases, 243 non-TB cases and 14 uncategorised cases. Uncategorised cases were excluded, leaving samples from 274 patients for analysis. Clinical characteristics for the included samples are shown in Table 1. Pleural fluid was the most common sample type. The MPT64 test was performed on all samples, whereas the type and number of routine TB diagnostic tests performed on the samples varied. HIV status was unknown for the study participants.

Among the 97 retrospectively included formalin-fixed biopsies, 13 biopsies were included because they had been subjected to a TB specific n-PCR at the pathology laboratory at $\mathrm{HUH}$, due to histopathological findings suggestive of TB. These samples had not been sent for $\mathrm{TB}$ diagnostics at the microbiology laboratory and $\mathrm{TB}$ was not mentioned as a differential diagnosis on the request form. Four of 13 samples were n-PCR positive, and $\mathrm{TB}$ was thus, an unexpected finding in these cases.

\section{MPT64 test performance compared to routine TB diagnostics and Xpert ultra \\ Biopsy specimens}

Using the CRS, the sensitivity (95\% CI) of the MPT64 test in formalin-fixed biopsies was $37 \%$ (16-62), compared to 20\% (4-48), 37\% (16-62) and 50\% (23-77) for microscopy, PCR-based tests pooled and culture respectively (Tables 2 and 3). Against PCR (1-step PCR and n-PCR pooled) as a reference standard, the sensitivity of the MPT64 test was 71\% (5/7, 95\% CI 29-96). However, in PCR negative, culture positive biopsies $(n=6)$, the MPT64 test was negative in all samples. One of the 69 non-TB biopsies was MPT64 test positive, yielding a positive predicitive value of $88 \%$ (7/8 MPT64 test positive biopsies were from TB cases) and an excellent specificity of $99 \%$ (95\% CI 92-100). Granulomatous inflammation with necrosis, the most specific histopathological finding suggestive of TB, was present in 13/19 biopsies from TB cases and 13/69 non-TB biopsies (Table 4). This gives histopathology a sensitivity, specificity and positive predicitive value of $68 \%$ (43-87), 81\% (70-90) and 50\% (36-64) respectively against the CRS. Among biopsies from non-TB cases, 5 samples were bacteriologically confirmed NTM infections and another 3 samples came from patients with a probable, though not confirmed, NTM infection. The MPT64 test was negative inn all these samples.

\section{Fine needle-aspirates and fluid samples}

Abundant non-specific staining was observed in the cell smears prepared from FNAs, pus and fluid samples, and the MPT64 test performance was lower in these materials compared to the biopsies. Using the CRS, the sensitivity
Table 1 Characteristics of samples included (one sample per patient)

$\begin{array}{ll}\text { TB } \text { cases }^{1} & \text { non-TB cases } \\ n=31 & n=243\end{array}$

Sample material

Lymph node aspirates

Lymph node biopsies

Other biopsies

Pus

Pleural fluid

Ascites

Pericardial fluid

Synovial fluid

Other fluids

Number of samples per patient ${ }^{2}$

\begin{tabular}{|c|c|c|c|}
\hline 1 sample & & 24 & 200 \\
\hline 2 samples & & 6 & 33 \\
\hline 3 samples & & 0 & 6 \\
\hline 4 samples & & 1 & 3 \\
\hline 5 samples & & 0 & 1 \\
\hline & Biopsies & $\begin{array}{l}\text { Fine-needle } \\
\text { aspirate }\end{array}$ & $\begin{array}{l}\text { Puss and } \\
\text { fluid samples }\end{array}$ \\
\hline & $n=88$ & $n=11$ & $n=175$ \\
\hline $\begin{array}{l}\text { ample sent to both } \\
\text { hicrobiology and } \\
\text { athology laboratories }\end{array}$ & 68 & 9 & 83 \\
\hline hicroscopy ${ }^{3}$ & 59 & 9 & 43 \\
\hline -step PCR & 32 & 6 & 8 \\
\hline lested-PCR & 12 & 4 & 1 \\
\hline ulture & 68 & 9 & 83 \\
\hline $\begin{array}{l}\text { ample only sent to the } \\
\text { hicrobiology laboratory }\end{array}$ & N/A & $N / A$ & 92 \\
\hline Иicroscopy & $\mathrm{N} / \mathrm{A}$ & $\mathrm{N} / \mathrm{A}$ & 51 \\
\hline -step PCR & N/A & N/A & 12 \\
\hline ulture & N/A & N/A & 92 \\
\hline $\begin{array}{l}\text { ample only sent to the } \\
\text { athology laboratory }\end{array}$ & 20 & 2 & N/A \\
\hline Microscopy & 4 & 0 & N/A \\
\hline lested-PCR & 17 & 1 & N/A \\
\hline
\end{tabular}

Abbreviations: TB, tuberculosis; $\mathrm{PCR}$, polymerase chain reaction, N/A, not applicable ${ }^{1}$ Includes 5 clinically diagnosed patients

${ }^{2}$ Number of samples per patient included at different time points. Patients with the same material from the same site $(n=27)$, different material from the same site $(n=13)$, material from different locations $(n=6)$, material from different locations, multiple samples collected from some of these locations $(n=4)$ ${ }^{3}$ Microscopy performed at pathology and/or microbiology laboratory. A sample with discordant microscopy results between the laboratories $(n=2)$ is registered as positive

and specificity of the MPT64 test in lymph node FNAs was $29 \%$ (95\% CI 4-71) and 50\% (95\% CI 7-93) respectively. Cytopathological findings suggestive of TB had low 
Table 2 Results of routine TB diagnostic tests, Xpert Ultra and the MPT64 test performed on samples

\begin{tabular}{|c|c|c|c|c|c|c|c|}
\hline & \multirow[b]{2}{*}{ Number of cases } & \multicolumn{6}{|c|}{ Positive samples/total samples (\%) } \\
\hline & & Micro-scopy ${ }^{a}$ & Culture & 1-step PCR & Nested-PCR & Xpert Ultra & MPT64 test \\
\hline \multicolumn{8}{|l|}{ TB cases } \\
\hline All samples & 31 & $6 / 27(22)$ & $16 / 26(62)$ & 6/18 (33) & $7 / 11(64)$ & 4/7 (57) & 10/31 (32) \\
\hline Lymph node biopsies & 7 & $1 / 6(17)$ & 2/6 (33) & $0 / 5(0)$ & $2 / 2(100)$ & N/A & $3 / 7(43)$ \\
\hline Other biopsies & 12 & $2 / 9(22)$ & $5 / 8(63)$ & $1 / 7(14)$ & $4 / 6(67)$ & N/A & 4/12 (33) \\
\hline Pus samples & 2 & $0 / 2(0)$ & $1 / 2(50)$ & $1 / 1(100)$ & N/A & $1 / 2(50)$ & $1 / 2(50)$ \\
\hline Lymph node aspirates & 7 & $3 / 7(43)$ & $5 / 7(71)$ & 4/4 (100) & $1 / 3(33)$ & $3 / 4(75)$ & 2/7 (29) \\
\hline Fluid samples & 3 & $0 / 3(0)$ & $3 / 3(100)$ & 0/1 (0) & N/A & 0/1 (0) & $0 / 3(0)$ \\
\hline Confirmed TB case -confirmed sample & $22^{\mathrm{b}}$ & $5 / 19(26)$ & 16/17 (94) & $6 / 13(46)$ & $7 / 9(78)$ & $4 / 5(80)$ & 8/22 (36) \\
\hline Culture positive sample & 16 & $4 / 16(25)$ & $16 / 16(100)$ & $5 / 12(42)$ & $2 / 4(50)$ & $4 / 5(80)$ & $3 / 16(19)$ \\
\hline Culture positive, $\mathrm{PCR}$ positive sample & 6 & $3 / 6(50)$ & $6 / 6(100)$ & $5 / 5(100)$ & $2 / 2(100)$ & $3 / 3(100)$ & $3 / 6(50)$ \\
\hline Culture positive, PCR negative sample & 8 & $1 / 8(13)$ & $8 / 8(100)$ & $0 / 7(0)$ & $0 / 2(0)$ & $1 / 2(50)$ & $0 / 8(0)$ \\
\hline 1-step PCR positive sample & 6 & $4 / 6(67)$ & $5 / 6(83)$ & $6 / 6(100)$ & 1/1 (100) & $3 / 3(100)$ & $4 / 6(67)$ \\
\hline Nested-PCR positive sample & 7 & $1 / 4(25)$ & $2 / 2(100)$ & $1 / 1(100)$ & 7/7 (100) & $1 / 1(100)$ & $5 / 7(71)$ \\
\hline Confirmed TB case -unconfirmed sample & 4 & 0/3 (0) & 0/4 (0) & $0 / 3(0)$ & N/A & N/A & 2/4 (50) \\
\hline Clinically diagnosed TB case & 5 & $1 / 5(20)$ & $0 / 5(0)$ & $0 / 2(0)$ & $0 / 2(0)$ & 0/2 (0) & $0 / 5(0)$ \\
\hline \multicolumn{8}{|l|}{ Non-TB cases } \\
\hline All samples & 243 & 7/139 (5) & 0/226 (0) & $0 / 40(0)$ & 0/24 (0) & $1 / 41(2)$ & $39 / 243(16)$ \\
\hline Lymph node biopsies & $17^{c}$ & $1 / 13(8)$ & 0/14 (0) & $0 / 4(0)$ & 0/4 (0) & 0/2 (0) & $0 / 17(0)$ \\
\hline Other biopsies & $52^{d}$ & $2 / 35(6)$ & 0/40 (0) & 0/16 (0) & 0/17 (0) & 1/8 (13) & $1 / 52(2)$ \\
\hline Pus samples & $13^{e}$ & 3/13 (23) & $0 / 13(0)$ & 0/4 (0) & 0/1 (0) & 0/8 (0) & 4/13 (31) \\
\hline Lymph node aspirates & $4^{f}$ & $1 / 2(50)$ & $0 / 2(0)$ & $0 / 2(0)$ & $0 / 2(0)$ & 0/1 (0) & $2 / 4(50)$ \\
\hline Fluid samples & 157 & $0 / 76(0)$ & $0 / 157(0)$ & $0 / 14(0)$ & N/A & $0 / 22(0)$ & $32 / 157(20)$ \\
\hline
\end{tabular}

Abbreviations: TB, tuberculosis; PCR, polymerase chain reaction

${ }^{a}$ Microscopy performed at microbiology laboratory and/or pathology laboratory. A sample with discordant microscopy results between the laboratories $(n=2)$ is registered as microscopy positive

${ }^{\mathrm{b}}$ One of the nested-PCR positive patients was not started on TB treatment, and only followed by controls

${ }^{\mathrm{C}} 6$ cases of NTM infection (2 NTM culture positive, 1 NTM specific PCR positive, 3 given a clinical diagnosis)

$\mathrm{d}_{2}$ cases of NTM infection (2 NTM specific PCR positive)

$\mathrm{e}_{3}$ cases of NTM infection ( 3 microscopy and NTM culture positive)

${ }^{f} 1$ case of NTM infection (microscopy, NTM culture and NTM specific PCR positive. This case was also MPT64 positive)

sensitivity and specificity for diagnosing TB. In pus and fluid samples, the sensitivity of all tests methods was difficult to evaluate due to few TB cases. All three pleural fluids from TB cases were culture positive and negative with all other tests. Two pus samples from TB cases were included. One was microscopy negative and positive with culture, 1-step PCR, Xpert Ultra and the MPT64 test, whereas the other sample was negative with all tests. Many non-TB pus and fluid samples were interpreted as weakly positive $(n=33)$ or positive $(n=3)$, and the specificity of the MPT64 test was $80 \%$ (95\% CI 72-86) and 69\% (95\% CI 39-91) in fluid and pus samples, respectively.

Head-to-head comparison of various diagnostic methods As the number of TB diagnostic tests performed on the samples varied greatly, the diagnostic performance of the different tests was also evaluated based on head-tohead comparisons (Table 5). There was no difference in the overall test performance between microscopy and the MPT64 test, which both detected the same number of TB cases as 1-step PCR, and fewer TB cases than nPCR, Xpert Ultra and culture. Further, the subgroup comparisons of culture, 1-step PCR, microscopy and the MPT64 test showed that some samples were positive in one test and negative in the other and vice versa. The MPT64 test was positive in 4 microscopy negative samples, 21 -step PCR negative samples and 3 culture negative samples, indicating added value of combining various $\mathrm{TB}$ diagnostic tests.

\section{Discussion}

This is the first study in which the MPT64 test, an immunochemistry-based test for diagnosing EPTB, has been implemented in parallel to routine TB diagnostics in a low TB prevalence country with a high-resource health care system. Using a CRS that included clinically 
Table 3 Test accuracy for various routine diagnostic tests, Xpert Ultra and the MPT64 test using a composite reference standard

\begin{tabular}{|c|c|c|c|c|}
\hline & $\begin{array}{l}\text { Test performed on } \\
\text { number of samples }\end{array}$ & $\begin{array}{l}\text { Sensitivity \% } \\
(95 \% \mathrm{Cl})\end{array}$ & $\begin{array}{l}\text { Specificity \% } \\
(95 \% \mathrm{Cl})\end{array}$ & $\begin{array}{l}\text { Overall } \\
\text { accuracy } \\
\%\end{array}$ \\
\hline \multicolumn{5}{|l|}{ All samples $(n=274)$} \\
\hline Microscopy & 166 & $22(9-42)$ & 95 (90-98) & 83 \\
\hline Culture & 252 & $62(41-80)$ & $100(98-100)$ & 96 \\
\hline PCR (1-step PCR and n-PCR pooled) & 87 & $44(26-65)$ & $100(94-100)$ & 83 \\
\hline Xpert Ultra & 48 & $57(18-90)$ & $98(87-100)$ & 92 \\
\hline MPT64 test & 274 & $32(17-51)$ & $84(79-88)$ & 78 \\
\hline \multicolumn{5}{|l|}{ Lymph node biopsies $(n=24)$} \\
\hline Microscopy & 19 & $17(0-64)$ & $92(64-100)$ & 68 \\
\hline Culture & 20 & $33(4-78)$ & $100(77-100)$ & 80 \\
\hline PCR (1-step PCR and n-PCR pooled) & 15 & $29(4-71)$ & $100(63-100)$ & 67 \\
\hline Xpert Ultra & 2 & N/A & $100(16-100)$ & - \\
\hline MPT64 test & 24 & $43(10-82)$ & $100(80-100)$ & 83 \\
\hline \multicolumn{5}{|l|}{ All biopsies $(n=88)$} \\
\hline Microscopy & 63 & $20(4-48)$ & $94(83-99)$ & 76 \\
\hline Culture & 68 & $50(23-77)$ & $100(93-100)$ & 90 \\
\hline PCR (1-step PCR and n-PCR pooled) & 57 & $37(16-62)$ & $100(91-100)$ & 79 \\
\hline Xpert Ultra & 10 & N/A & $90(56-100)$ & - \\
\hline MPT64 test & 88 & $37(16-62)$ & $99(92-100)$ & 85 \\
\hline \multicolumn{5}{|l|}{ Lymph node aspirates $(\mathrm{n}=11)$} \\
\hline Microscopy & 9 & $43(10-82)$ & $50(1-99)$ & 44 \\
\hline Culture & 9 & $71(29-96)$ & $100(16-100)$ & 78 \\
\hline PCR (1-step PCR and n-PCR pooled) & 9 & $67(22-96)$ & $100(29-100)$ & 78 \\
\hline Xpert Ultra & 5 & 75 (19-99) & $100(3-100)$ & 80 \\
\hline MPT64 test & 11 & $29(4-71)$ & $50(7-93)$ & 36 \\
\hline \multicolumn{5}{|l|}{ Pus samples $(n=15)$} \\
\hline Microscopy & 15 & $0(0-84)$ & $77(46-95)$ & 67 \\
\hline Culture & 15 & $50(1-99)$ & $100(75-100)$ & 93 \\
\hline PCR (1-step PCR and n-PCR pooled) & 6 & $100(3-100)$ & $100(48-100)$ & 100 \\
\hline Xpert Ultra & 10 & $50(1-99)$ & $100(63-100)$ & 90 \\
\hline MPT64 test & 15 & $50(1-99)$ & 69 (39-91) & 67 \\
\hline \multicolumn{5}{|l|}{ Fluid samples $(n=160)$} \\
\hline Microscopy & 79 & $0(0-71)$ & $100(95-100)$ & 96 \\
\hline Culture & 160 & $100(29-100)$ & $100(98-100)$ & 100 \\
\hline PCR (1-step PCR and n-PCR pooled) & 15 & $0(0-98)$ & $100(77-100)$ & 93 \\
\hline Xpert Ultra & 23 & $0(0-98)$ & $100(85-100)$ & 96 \\
\hline MPT64 test & 160 & $0(0-71)$ & $80(72-86)$ & 78 \\
\hline
\end{tabular}

Abbreviations: $\mathrm{PCR}$, polymerase chain reaction; $\mathrm{Cl}$, confidence interval

diagnosed TB cases, the test had a sensitvity (95\% CI) of $37 \%$ (16-62) in formalin-fixed biopsies, compared to $37 \%(16-62)$ and $50 \%(23-77)$ for PCR-based tests pooled and culture, respectively. The specificity of the test was excellent $(99,95 \%$ CI 92-100) in formalin-fixed biopsies. In cell smears prepared from FNAs, pus and fluid samples, the test performance was low. Culture was found to be the most sensitive method for diagnosing TB in the study, with a disadvantage of long turnaround time. The study revealed that in this low TB incidence setting, many EPTB cases are incidentally detected based on histopathological findings in biopsy specimens that 
Table 4 Cyto/histopathological findings in biopsy and fine-needle aspirate samples

\begin{tabular}{|c|c|c|c|c|c|}
\hline & \multicolumn{4}{|l|}{ TB cases } & \multirow[t]{2}{*}{ Non-TB cases } \\
\hline & Confirmed sample & Unconfirmed sample & Clinically diagnosed sample & Total TB cases & \\
\hline Histomorphology, biopsies ( $n=88$ ) & $n=13$ & $n=3$ & $n=3$ & $n=19$ & $n=69$ \\
\hline $\begin{array}{l}\text { Granulomatous inflammation } \\
\text { with necrosis }\end{array}$ & 9 & 3 & 1 & 13 & 13 \\
\hline $\begin{array}{l}\text { Granulomatous inflammation } \\
\text { without necrosis }\end{array}$ & 1 & & 2 & 3 & 13 \\
\hline $\begin{array}{l}\text { Subacute or chronic } \\
\text { inflammation and necrosis }\end{array}$ & 3 & & & 3 & 4 \\
\hline Abundant necrosis & & & & & 3 \\
\hline $\begin{array}{l}\text { Subacute or chronic } \\
\text { inflammation }\end{array}$ & & & & & 22 \\
\hline Acute inflammation & & & & & 3 \\
\hline Malignant tumor & & & & & 4 \\
\hline Benign tumor & & & & & 2 \\
\hline Fibrosis & & & & & 1 \\
\hline Foreign body granuloma & & & & & 1 \\
\hline Lymph node hyperplasia & & & & & 1 \\
\hline Mesothelial proliferation & & & & & 1 \\
\hline No pathology & & & & & 1 \\
\hline Cytomorphology, FNAs $(n=11)$ & $n=5$ & $n=1$ & $\mathrm{n}=1$ & $n=7$ & $n=4$ \\
\hline $\begin{array}{l}\text { Granulomatous inflammation } \\
\text { with necrosis }\end{array}$ & & & 1 & 1 & 1 \\
\hline Abundant necrosis & 3 & & & 3 & 1 \\
\hline Fluid background, mostly RBCs & 1 & & & 1 & 1 \\
\hline Subacute or chronic inflammation & 1 & & & 1 & 1 \\
\hline Acute inflammation & & 1 & & 1 & \\
\hline
\end{tabular}

Abbreviations: TB, tuberculosis; FNA, fine-needle aspirate

have not been sent for culture in parallel. Histopathological findings alone cannot confirm a TB diagnosis, and in these cases, the MPT64 test can supplement other rapid tests, including microscopy and n-PCR. This test is less prone to contamination than PCR and, in contrast to microscopy, can differentiate between MTBC and NTM infections. Thus, the MPT64 test may strengthen the TB diagnosis in a pathology laboratory in the absence of culture confirmation.

The MPT64 test performance was lower in the present study compared to previous studies [28-30,35]. Against a CRS, the overall sensitivity was $32 \%$ (95\% CI 17-51) for the MPT64 test, compared to $67-100 \%$ in previous studies [28-30,35]. The use of different composite reference standards and variable $\mathrm{TB}$ prevalence across the studies may contribute to this variation. All previous studies were conducted in high TB burden settings, in which a higher pre-test probability of $\mathrm{TB}$ combined with potentially more advanced stage of $\mathrm{TB}$ disease at the time of diagnosis, may lead to higher test sensitivity. Still, also when using culture as a reference standard, the overall MPT64 test sensitivity was lower (19, 95\% CI 4-46) compared to previous studies $(75-100 \%)[26,28,30,35$, 37]. This could partly be explained by different procedures for culture used across the studies. Apportioning of smaller sample volumes for culture and long transportation time to the TB laboratory, potentially reducing the viability of the bacilli, may have reduced the sensitivity of culture in previous studies [35]. In most of the previous studies, all samples were decontaminated and seeded onto only 1 tube of solid medium, whereas 2-8 culture tubes per sample were used for most samples in the present study, including culturing of material not treated with NALC- $\mathrm{NaOH}$ for many samples. These factors can lead to increased sensitivity of culture in our study, especially in paucibacillary specimens with non-uniform distribution of bacilli. Further, the use of different reference standards makes it challenging to compare the studies. For validation of the MPT64 test, n-PCR has been used as a reference standard in most previous studies, yielding a sensitivity of $72-100 \%$ [26-29, 31, 35]. In the present study, n-PCR was only performed on a subgroup of samples and could not be used for validation alone. However, when using n-PCR as a reference standard in this subgroup, the sensitivity of the MPT64 test was 71\% 
Table 5 Head-to-head comparison of different TB diagnostic tests, including Xpert Ultra and the MPT64 test among TB cases $^{1}$

\begin{tabular}{|c|c|c|c|c|c|c|c|c|c|c|}
\hline & $\begin{array}{l}\text { All TB CC } \\
n=31\end{array}$ & & & & & & & & & \\
\hline & $\begin{array}{l}\text { MPT64 } \\
\text { perform }\end{array}$ & & $\begin{array}{l}\text { Microscopy } \\
\text { performed }\end{array}$ & & $\begin{array}{l}\text { 1-step } \\
\text { perforn }\end{array}$ & & $\begin{array}{l}\text { Nested-PCR } \\
\text { performed }\end{array}$ & & $\begin{array}{l}\text { Culture } \\
\text { performed }\end{array}$ & \\
\hline & $n=31$ & & $n=27$ & & $n=18$ & & $n=11$ & & $n=26$ & \\
\hline & $\begin{array}{l}\text { MPT64 } \\
\text { test + }\end{array}$ & $\begin{array}{l}\text { MPT64 } \\
\text { test - }\end{array}$ & Micro-scopy + & Micro-scopy - & $\begin{array}{l}\text { 1-step } \\
\text { PCR+ }\end{array}$ & $\begin{array}{l}\text { 1-step } \\
\text { PCR- }\end{array}$ & Nested-PCR+ & Nested-PCR- & Culture + & Culture - \\
\hline & $n=10$ & $n=21$ & $n=6$ & $n=21$ & $n=6$ & $n=12$ & $n=7$ & $n=4$ & $n=16$ & $n=10$ \\
\hline Microscopy + & 2 & 4 & & & & & & & & \\
\hline Microscopy - & 4 & 17 & & & & & & & & \\
\hline 1-step PCR + & 4 & 2 & 4 & 2 & & & & & & \\
\hline 1-step PCR - & 2 & 10 & 2 & 9 & & & & & & \\
\hline Nested-PCR + & 5 & 2 & 1 & 3 & 1 & 0 & & & & \\
\hline Nested-PCR - & 0 & 4 & 0 & 4 & 0 & 1 & & & & \\
\hline Culture + & 3 & 13 & 4 & 12 & 5 & 7 & 2 & 2 & & \\
\hline Culture - & 3 & 7 & 2 & 7 & 1 & 5 & 0 & 2 & & \\
\hline Xpert Ultra + & 2 & 2 & 2 & 2 & 3 & 0 & 1 & 1 & 4 & 0 \\
\hline Xpert UItra - & 0 & 3 & 0 & 3 & 0 & 1 & 0 & 1 & 1 & 2 \\
\hline
\end{tabular}

Abbreviations: TB, tuberculosis; PCR, polymerase chain reaction

${ }^{1}$ TB cases include both microbiologically confirmed TB cases $(n=26)$ and clinically diagnosed TB cases $(n=5)$

(95\% CI 29-96). This is close to previous findings. Moreover, all culture positive samples were n-PCR positive in previous studies, whereas the present study included several culture positive, but PCR negative samples $(n=8)$. The MPT64 test was negative in all these culture positive, PCR negative samples. Assuming that culture positive, PCR negative samples have a lower bacterial load than culture positive, PCR positive samples, these results indicate that the MPT64 test is not sensitive enough to detect samples with very low bacterial load. However, the long turnout time of culture does not help clinicians to make a timely diagnosis. Further, although culture performed under optimal conditions is the most sensitive method for diagnosing EPTB in the present study, TB culture facilities are not available in most TB endemic areas, in which TB diagnostics are most needed.

The specificity of the MPT64 test in biopsy specimens was very high and comparable to results observed in previous studies, whereas the specificity in cell smears prepared from FNAs, pus and fluid samples was lower. In lymph node FNAs, the specificity was only $50 \%$, However, this was based on only two MPT64 test positive non-TB cases of a total of four non-TB cases, which gives low power to the estimate. In pus and fluid samples, non-specific false positive staining was observed in a large proprotion of the smears and made interpretation challenging. The non-specific staining may have been caused by suboptimal sample handling at the microbiology laboratories where samples could be stored cold for more than one day before preparation of smears, as indicated by cell lysis in many samples. Long storage time may have affected the antigen integrity and increased nonspecific binding. In contrast, smears in previous studies were prepared immediately after sample collection.

The low specificity of the test in cell smears has a greater impact in this low prevalence setting compared to a high prevalence setting because more false positive cases and unecessary treatment must be accepted for every true positive case detected. Thus, the results of the present study indicate that the MPT64 test is not useful for diagnosis of EPTB in cell smears. In biopsy specimens, on the other hand, the test was highly specific. It was negative in clinically relevant non-TB samples with various types of inflammation and in all samples from patients with NTM infections. NTM infection is an important differential diagnosis to EPTB, as $31 \%$ of the microbiologically confirmed mycobacterial infections were NTM in the present study.

There are limitations to the study. The low number of TB cases gives low power to sensitivity estimates.. Further, the exlusion of culture and PCR negative samples because information about clinical TB diagnosis was not available (Fig. 1), in addition to the exclusion of biopsies with a histopathological diagnosis other than TB or no pathological findings, leads to a selection bias in favour of samples with a higher pre-test probability of $\mathrm{TB}$, which could affect specificity estimates. As the study was designed to evaluate the MPT64 test performance in a routine setting, we did not intervene in sample handling or TB diagnostic algorithms at the inclusion sites, leading to many suboptimally prepared samples for the MPT64 test. Samples from patients with presumptive 
EPTB were often not sent for TB diagnostics both to microbiology and pathology laboratories, as would have been expected according to good clinical practice. Not only may this lead to diagnostic delay since the available diagnostic tools are not fully utilized in difficult-todiagnose cases, but it also makes it difficult to compare test performance in the present study because the types and number of tests performed per sample varied greatly. Hence, more controlled validation studies with larger cohorts are needed to fully asses MPT64 test performance in a low TB incidence high-resource setting.

\section{Conclusions}

The diagnosis of EPTB is challenging in a high-resource, low-TB incidence country. The awareness of TB is often low and routine $\mathrm{TB}$ diagnostic tests are not able to identify all EPTB cases. The MPT64 antigen detection test has a good positive predictive value and an excellent specificity in formalin-fixed biopsies and is implementable in pathology laboratories. In the absence of culture, the MPT64 test may contribute to strengthen the TB diagnosis in formalin-fixed biopsies when used in combination with microscopy and PCR-based tests, and thus, has an added value in TB diagnostics in this setting.

\section{Abbreviations}

AFB: Acid fast bacilli; CRS: Composite reference standard;

EPTB: Extrapulmonary tuberculosis; FNA: Fine needle aspirate; HUH: Haukeland University Hospital; MTBC: Mycobacterium tuberculosis complex; n-PCR: Nested-PCR; NTM: Non-tuberculosis mycobacteria; OUH: Oslo University Hospital; SUH: Stavanger University Hospital; TB: Tuberculosis; WHO: World Health Organisation

\section{Acknowledgements}

We thank Edith Marianne Fick, University of Bergen/Haukeland University Hospital, and Sonja Ljostvedt, University of Bergen, for technical assistance; Ranja Christiansen, Haukeland University Hospital, for screening the samples; Elling Ulvestad, Haima Mylvaganam, Dag Harald Skutlaberg and Ba Ngoc Vu, Department of Microbiology, Haukeland University Hospital, Ulf Christian Lidstedt, Department of Medical Microbiology, Stavanger University Hospital, and Fredrik Müller og Finn Bjørnar Jakobsen, Department of Microbiology, Oslo University Hospital, for facilitating and contributing to data collection.

\section{Authors' contributions}

TM conceptualised the study. Study design and methodology was developed by TM, LS, HW, MDJ and IMH. AMDR, IS, HS, PJ and IMH collected data. LS and TM interpreted and validated the results of the study. IMH performed formal analysis of data. IMH and TM wrote the original draft. IMH, LS, HS, AMD, IS, PJ, MDJ, HW and TM reviewed and edited the manuscript. All authors have read and approved the final manuscript.

\section{Funding}

This work was partly supported by the Research Council of Norway through the Global Health and Vaccination Programme [project number 234457]. This project is part of the EDCTP2 programme supported by the European Union. The funders had no role in study design, data collection and analysis, decision to publish, or preparation of the manuscript.

\section{Availability of data and materials}

The datasets generated and analysed during the current study are available from the corresponding author on reasonable request.

\section{Ethics approval and consent to participate}

The study was approved by the Regional Committee for Medical and Health Research Ethics of Western Norway (REK Vest) (2014/46/REK vest). An exemption from informed consent from the patients was granted as the study only included residual material from samples sent for TB diagnostics in the clinical setting.

\section{Consent for publication}

Not applicable

\section{Competing interests}

The authors declare that they have no competing interests.

\section{Author details}

${ }^{1}$ Centre for International Health, Department of Global Public Health and Primary Care, University of Bergen, Bergen, Norway. ${ }^{2}$ Department of Clinical Science, University of Bergen, Bergen, Norway. ${ }^{3}$ Department of Clinical Medicine, University of Bergen, Bergen, Norway. ${ }^{4}$ Department of Pathology, Haukeland University Hospital, Oslo, Norway. ${ }^{5}$ Department of Medical Microbiology, Stavanger University Hospital, Stavanger, Norway. ${ }^{6}$ Department of Infectious Diseases, Oslo University Hospital, Oslo, Norway. ${ }^{7}$ Institute of Clinical Medicine, University of Oslo, Oslo, Norway. ${ }^{8}$ Department of Microbiology, Haukeland University Hospital, Bergen, Norway. ${ }^{9}$ Department of Pathology, Oslo University Hospital, Oslo, Norway. ${ }^{10}$ Department of Thoracic Medicine, Haukeland University Hospital, Bergen, Norway.

Received: 5 October 2019 Accepted: 6 February 2020

Published online: 12 February 2020

\section{References}

1. World Health Organization. Global tuberculosis report 2018. 2018 [cited 2018 Dec 10]. Available from: https://www.who.int/tb/publications/global_ report/en/.

2. Folkehelseinstituttet. Tuberkulose i Norge 2017. 2018 [cited 2018 Dec 5]. Available from: https://www.fhi.no/publ/2018/tuberkulose-i-norge-2017\%2 D\%2D-med-behandlingsresultater-for-2016.-arsrapport/.

3. Kruijshaar ME, Abubakar I. Increase in extrapulmonary tuberculosis in England and Wales 1999-2006. Thorax. 2009;64(12):1090.

4. Enviroment NIfPHat. Tuberculosis in the Netherlands 2017: Surveillance Rep 2018.

5. Te Beek LA, Van Der Werf MJ, Richter C, Borgdorff MW. Extrapulmonary tuberculosis by nationality, the Netherlands, 1993-2001. Emerg Infect Dis. 2006;12(9):1375.

6. Sandgren A, Hollo V, Van der Werf M. Extrapulmonary tuberculosis in the European union and European economic area, 2002 to 2011. Eurosurveillance. 2013;18(12):20431.

7. Peto HM, Pratt RH, Harrington TA, LoBue PA, Armstrong LR. Epidemiology of extrapulmonary tuberculosis in the United States, 1993-2006. Clin Infect Dis. 2009:49(9):1350-7.

8. European Centre for Disease Prevention and Control/WHO Regional Office for Europe. Tuberculosis surveillance and monitoring in Europe 2018-2016 data. 2018 [cited 2019 Jan 19]. Available from: https://ecdc.europa.eu/sites/ portal/files/documents/ecdc-tuberculosis-surveillancemonitoring-Europe-201 8-19mar2018.pdf.

9. Dinnes J, Deeks J, Kunst H, Gibson A, Cummins E, Waugh N, et al. A systematic review of rapid diagnostic tests for the detection of tuberculosis infection. 2007.

10. Kohli M, Schiller I, Dendukuri N, Dheda K, Denkinger CM, Schumacher SG, et al. Xpert ${ }^{\circledR}$ MTB/RIF assay for extrapulmonary tuberculosis and rifampicin resistance. Cochrane Database Syst Rev. 2018;8.

11. World Health Organization. Xpert MTB/RIF implementation manual. Technical and operational "how-to": practical considerations. 2014 [cited 2018 Dec 5]. Available from: https://www.who.int/tb/publications/xpert_ implem_manual/en/.

12. Dorman SE, Schumacher SG, Alland D, Nabeta P, Armstrong DT, King B, et al. Xpert MTB/RIF ultra for detection of Mycobacterium tuberculosis and rifampicin resistance: a prospective multicentre diagnostic accuracy study. Lancet Infect Dis. 2018;18(1):76-84.

13. Bahr NC, Nuwagira E, Evans EE, Cresswell FV, Bystrom PV, Byamukama A, et al. Diagnostic accuracy of Xpert MTB/RIF ultra for tuberculous meningitis 
in HIV-infected adults: a prospective cohort study. Lancet Infect Dis. 2018; 18(1):68-75.

14. Bisognin F, Lombardi G, Lombardo D, Re MC, Dal MP. Improvement of Mycobacterium tuberculosis detection by Xpert MTB/RIF ultra: a head-tohead comparison on Xpert-negative samples. PLoS One. 2018;13(8): e0201934.

15. Atherton RR, Cresswell FV, Ellis J, Skipper C, Tadeo KK, Mugumya G, et al. Detection of Mycobacterium tuberculosis in urine by Xpert MTB/RIF ultra: a useful adjunctive diagnostic tool in HIV-associated tuberculosis. Int J Infect Dis. 2018;75:92-4.

16. Perez-Risco D, Rodriguez-Temporal D, Valledor-Sanchez I, Alcaide F. Evaluation of the Xpert MTB/RIF ultra assay for direct detection of Mycobacterium tuberculosis complex in smear-negative Extrapulmonary samples. J Clin Microbiol. 2018:56(9):e00659-18.

17. Wu X, Tan G, Gao R, Yao L, Bi D, Guo Y, et al. Assessment of the Xpert MTB/ RIF ultra assay on rapid diagnosis of extrapulmonary tuberculosis. Int $J$ Infect Dis. 2019:81:91-6.

18. Chin JH, Musubire AK, Morgan N, Pellinen J, Grossman S, Bhatt JM, et al. Xpert MTB/RIF ultra for the detection of Mycobacterium tuberculosis in cerebrospinal fluid. J Clin Microbiol. 2019.

19. López-Varela E, García-Basteiro AL, Santiago B, Wagner D, van Ingen J, Kampmann B. Non-tuberculous mycobacteria in children: muddying the waters of tuberculosis diagnosis. Lancet Respir Med. 2015;3(3):244-56.

20. Marras TK, Chedore P, Ying AM, Jamieson F. Isolation prevalence of pulmonary non-tuberculous mycobacteria in Ontario 1997-2003. Thorax. 2007.

21. Lacroix A, Piau C, Lanotte P, Carricajo A, Guillouzouic A, Peuchant O, et al. Emergence of Nontuberculous mycobacterial lymphadenitis in children after the discontinuation of mandatory Bacillus Calmette and GuÉrin immunization in France. Pediatr Infect Dis J. 2018;37(10):e257-e60.

22. Kontturi A, Soini H, Ollgren J, Salo E. Increase in Childhood Nontuberculous Mycobacterial Infections After Bacille Calmette-Guérin Coverage Drop: A Nationwide, Population-Based Retrospective Study, Finland, 1995-2016. Clinical Infectious Diseases. 2018:ciy241.

23. Storla DG, Yimer S, Bjune GA. A systematic review of delay in the diagnosis and treatment of tuberculosis. BMC Public Health. 2008;8(1):15.

24. Farah MG, Rygh JH, Steen TW, Selmer R, Heldal E, Bjune G. Patient and health care system delays in the start of tuberculosis treatment in Norway. BMC Infect Dis. 2006;6(1):33.

25. Jørstad MD, A mus J, Marijani M, Sviland L, Mustafa T. Diagnostic delay in extrapulmonary tuberculosis and impact on patient morbidity: a study from Zanzibar. PLoS One. 2018;13(9):e0203593.

26. Mustafa T, Wiker HG, Mfinanga SG, Mørkve O, Sviland L. Immunohistochemistry using a Mycobacterium tuberculosis complex specific antibody for improved diagnosis of tuberculous lymphadenitis. Mod Pathol. 2006;19(12):1606-14.

27. Purohit MR, Mustafa T, Wiker HG, Mørkve O, Sviland L. Immunohistochemical diagnosis of abdominal and lymph node tuberculosis by detecting Mycobacterium tuberculosis complex specific antigen MPT64. Diagn Pathol. 2007;2:36.

28. Purohit MR, Mustafa T, Wiker HG, Sviland L. Rapid diagnosis of tuberculosis in aspirate, effusions, and cerebrospinal fluid by immunocytochemical detection of Mycobacterium tuberculosis complex specific antigen MPT64. Diagn Cytopathol. 2012;40(9):782-91.

29. Baba K, Dyrhol-Riise AM, Sviland L, Langeland N, Hoosen AA, Wiker HG, et al. Rapid and specific diagnosis of tuberculous pleuritis with immunohistochemistry by detecting Mycobacterium tuberculosis complex specific antigen MPT64 in patients from a HIV endemic area. Appl Immunohistochem Mol Morphol. 2008;16(6):554-61.

30. Tadele A, Beyene D, Hussein J, Gemechu T, Birhanu A, Mustafa T, et al. Immunocytochemical detection of Mycobacterium tuberculosis complex specific antigen, MPT64, improves diagnosis of tuberculous lymphadenitis and tuberculous pleuritis. BMC Infect Dis. 2014;14(1):585.

31. Purohit MR, Sviland L, Wiker $H$, Mustafa T. Rapid and specific diagnosis of extrapulmonary tuberculosis by immunostaining of tissues and aspirates with anti-MPT64. Appl Immunohistochem Mol Morphol. 2017;25(4):282-8.

32. Harboe M, Nagai S, Patarroyo ME, Torres M, Ramirez C, Cruz N. Properties of proteins MPB64, MPB70, and MPB80 of Mycobacterium bovis BCG. Infect Immun. 1986;52(1):293-302.

33. Elhay MJ, Oettinger T, Andersen P. Delayed-type hypersensitivity responses to ESAT-6 and MPT64 from Mycobacterium tuberculosis in the Guinea pig. Infect Immun. 1998;66(7):3454-6.
34. Mahairas GG, Sabo PJ, Hickey MJ, Singh DC, Stover CK. Molecular analysis of genetic differences between Mycobacterium bovis BCG and virulent M. bovis. J Bacteriol. 1996;178(5):1274-82.

35. Jørstad MD, Marijani M, Dyrhol-Riise AM, Sviland L, Mustafa T. MPT64 antigen detection test improves routine diagnosis of extrapulmonary tuberculosis in a low-resource setting: a study from the tertiary care hospital in Zanzibar. PLoS One. 2018;13(5):e0196723.

36. Greenwood N, Fox H. A comparison of methods for staining tubercle bacilli in histological sections. J Clin Pathol. 1973;26(4):253-7.

37. Purohit MR, Mustafa T, Sviland L. Detection of Mycobacterium tuberculosis by polymerase chain reaction with DNA eluted from aspirate smears of tuberculous lymphadenitis. Diagn Mol Pathol. 2008;17(3):174-8.

\section{Publisher's Note}

Springer Nature remains neutral with regard to jurisdictional claims in published maps and institutional affiliations.

\section{Ready to submit your research? Choose BMC and benefit from:}

- fast, convenient online submission

- thorough peer review by experienced researchers in your field

- rapid publication on acceptance

- support for research data, including large and complex data types

- gold Open Access which fosters wider collaboration and increased citations

- maximum visibility for your research: over $100 \mathrm{M}$ website views per year

At BMC, research is always in progress.

Learn more biomedcentral.com/submissions 